\title{
Implementation of LS, MMSE and SAGE Channel Estimators for Mobile MIMO-OFDM
}

\author{
Johanna Ketonen and Markku Juntti \\ Centre for Wireless Communications \\ University of Oulu, Finland \\ \{johanna.ketonen, markku.juntti\}@ee.oulu.fi
}

\author{
Jari Ylioinas \\ Nokia Siemens Networks \\ Oulu, Finland \\ jari.ylioinas@nsn.com
}

\author{
Joseph R. Cavallaro \\ Rice University \\ Houston, Texas \\ cavallar@rice.edu
}

\begin{abstract}
The use of decision directed (DD) channel estimation in a multiple-input multiple-output (MIMO) orthogonal frequency division multiplexing (OFDM) downlink receiver is studied in this paper. The 3GPP long term evolution (LTE) based pilot structure is used as a benchmark. The space-alternating generalized expectation-maximization (SAGE) algorithm is used to improve the performance from that of the pilot symbol based least-squares (LS) channel estimator. The DD channel estimation improves the performance with high user velocities, where the pilot symbol density is not sufficient. Minimum mean square error (MMSE) filtering can also be used in estimating the channel in between pilot symbols. The DD channel estimation can be used to reduce the pilot overhead without any performance degradation by transmitting data instead of pilot symbols. The pilot overhead is reduced to a third of the LTE pilot overhead, obtaining a ten percent increase in throughput. The pilot based LS, MMSE and the SAGE channel estimators are implemented and the performance-complexity trade-offs are studied.
\end{abstract}

\section{INTRODUCTION}

Multiple-input multiple-output (MIMO) systems offer an increase in capacity or diversity. Orthogonal frequency division multiplexing (OFDM) is a popular technique for wireless high data-rate transmission because it enables efficient use of the available bandwidth and a simple implementation. The combination of MIMO and OFDM is a popular wireless access scheme and it has been adopted in the third generation partnership project (3GPP) long term evolution (LTE) and LTE advanced (LTE-A) standards [1] as well as in the Worldwide Interoperability for Microwave Access (WiMAX) system.

The reference signals or pilot symbols used in channel estimation are placed in the OFDM time-frequency grid at certain intervals in the LTE system [1]. The interval may not be sufficiently short when the user velocity is high and the channel is fast fading. The high mobility scenario, which is included in the LTE-A requirements, calls for the use of spatial multiplexing when the channel state information (CSI) at the transmitter becomes outdated for transmission adaptation. Furthermore, the pilot overhead increases with the number of MIMO streams. It becomes more problematic as the number of antennas in the system increase. Additionally, channel estimation based on only pilot symbols does not utilize the channel information available in the data decisions. Decision

This research has been supported in part by Tekes, the Finnish Funding Agency for Technology and Innovation, Nokia, Nokia Siemens Networks, Renesas Mobile Europe, Elektrobit, Xilinx and Academy of Finland as well as the Nokia Foundation. directed (DD) channel estimation can be used to improve the performance by exploiting the information on the non-pilot symbols or to reduce the pilot overhead by transmitting data symbols instead of pilot symbols.

The least-squares (LS) method attempts to minimize the squared difference between the received signal and the known pilot symbols or the data decisions [2]. Maximum likelihood (ML) channel estimation is equivalent to LS estimation with additive white Gaussian noise when the number of pilot symbols is larger than the channel length [3]. Using the LS channel estimation with data decisions would incur a high complexity in the receiver due to the large matrix inversion. The recursive LS (RLS) algorithm can be used to enhance the channel estimation performance but it is most suitable for slow fading channels [4]. The expectation-maximization (EM) algorithm [5] can be used to calculate the maximum likelihood (ML) estimate iteratively, avoiding the matrix inversion. The space-alternating generalized expectation-maximization (SAGE) algorithm [6] provides faster convergence than the EM algorithms. Minimum mean square error (MMSE) filtering can be used in channel estimation to improve the performance by including the time domain (TD) and spatial correlation in the estimation [7],[8]. An implementation of an approximate linear MMSE channel estimator was presented in [9] and an implementation of a channel estimator for fading channels was discussed in [10]. The performance and the complexity in number of multiplications of the LS and SAGE algorithms were presented in [11] but the MMSE algorithm or implementations of the channel estimation algorithms were not included. Several algorithms for channel estimation in high velocity scenarios have been proposed. However, the actual implementation cost or a performance-complexity comparison of the algorithms has not been previously discussed. Thus, this is the scope of the paper.

In this paper, performance of the LS, MMSE and SAGE channel estimation algorithms is studied using the LTE pilot symbol structure [1] as a benchmark. Two throughput decreasing issues are addressed, namely the fast fading or high mobility scenario with insufficient pilot symbol density and the high pilot overheads from the MIMO pilot symbols. The SAGE channel estimator is used in the iterative receiver to improve the performance when the pilot symbol density is too low, i.e, in high velocity cases. MMSE filtering is also used in between pilot symbols to improve the channel estimates and the performance is compared to that of the SAGE estimator. 
The throughput can be increased by replacing some of the pilot symbols with data symbols and using the SAGE algorithm to compensate for the performance loss caused by the decreased pilot density. The architecture and implementation results in gate counts and power consumption for the pilot symbol based LS, MMSE and the DD SAGE channel estimators are presented for the $2 \times 2$ and $4 \times 4$ antenna systems. For a more energy efficient solution, a longer latency for the channel estimator is considered. The impact of generating a timely channel estimate for the detector on the performance and complexity is then discussed.

\section{SySTEM MODEL}

An OFDM based MIMO transmission system with $N$ transmit (TX) and $M$ receive (RX) antennas, where $N \leq M$, is considered in this paper. The LTE standard specifies a maximum of two separately encoded data streams [1]. A layered space-time architecture with horizontal encoding is applied, i.e, the two data streams are encoded and decoded separately. In the $4 \times 4$ antenna system, each of the two streams are multiplexed onto two antennas; the first stream is multiplexed onto the first and second antenna and the second stream onto the third and fourth antenna. The soft symbol estimates from the decoder are used in the channel estimation.

The received frequency domain (FD) signal vector $\mathbf{y}(n)$ on the $m_{R}$ th receive antenna at discrete time index $n$ after the discrete Fourier transform (DFT) can be described as

$$
\underline{\mathbf{y}}_{m_{R}}(n)=\mathbf{X}(n) \underline{\mathbf{F}} \mathbf{h}_{m_{R}}(n)+\mathbf{w}_{m_{R}}(n),
$$

where $\mathbf{X}(n)=\left[\mathbf{X}_{1}, \ldots, \mathbf{X}_{N}\right] \in \mathbb{C}^{P \times N P}$ is the transmitted signal over $P$ subcarriers and $N$ transmit antennas, $\mathbf{w}_{m_{R}} \in$ $\mathbb{C}^{P}$ contains identically distributed complex Gaussian noise, $\underline{\mathbf{F}}=\mathbf{I}_{N} \otimes \mathbf{F}$ is a $N P \times N L$ matrix from the DFT matrix with $[\mathbf{F}]_{u, s}=\frac{1}{\sqrt{P}} e^{-j 2 \pi u s / P}, u=0, \ldots, P-1, s=0, \ldots, L-1, L$ is the length of the channel impulse response and $\mathbf{h}_{m_{R}} \in \mathbb{C}^{N L}$ is the time domain channel vector from the transmit antennas to the $m_{R}$ th receive antenna. The entries of the diagonal matrix $\mathbf{X}_{m_{T}} \in \mathbb{C}^{P \times P}$ are from a complex quadrature amplitude modulation (QAM) constellation $\Omega$ and $|\Omega|=2^{Q}$, where $Q$ is the number of bits per symbol and $m_{T}=1, \ldots, N$ and $m_{R}=1, \ldots, M$.

The cell-specific reference signal or pilot symbols are transmitted in a resource element grid as in LTE [1]. Reference signals are transmitted in the first, second and fifth OFDM symbols. Nothing is transmitted on the other antenna ports when a reference signal is transmitted on one antenna port. The reference signals for each antenna port are mapped to every 6th resource element in frequency. Quadrature phase shift keying (QPSK) modulated reference signals are assumed. The pilot overhead in the $2 \times 2$ MIMO is roughly $9.5 \%$ and in the $4 \times 4$ MIMO $14 \%$.

\section{Channel Estimation}

The receiver structure is presented in Fig. 1. The LS channel estimator is used in calculating the channel estimates from pilot symbols. The received signal vector is transformed into frequency domain before the LS channel estimation. The
LS channel estimate can be filtered with an MMSE filter. The channel impulse response result from the LS or MMSE estimator has to be transformed into frequency domain for the detector with the second fast fourier transform (FFT). The DD SAGE channel estimator can be used in addition to the LS estimator. The pilot based LS estimator provides initial channel estimates for the SAGE. The soft symbols are calculated from the decoder outputs and are transformed into time domain for the SAGE channel estimator. The SAGE channel estimator also takes the time domain received signal as input.

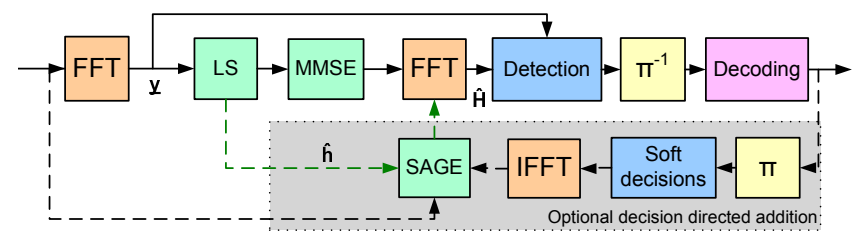

Fig. 1. The receiver structure.

\section{A. LS Channel Estimation}

The LS estimate of the channel can be calculated as

$$
\hat{\mathbf{h}}_{m_{R}}^{\mathrm{LS}}(n)=\left(\mathbf{F}^{\mathrm{H}} \mathbf{X}^{\mathrm{H}}(n) \mathbf{X}(n) \mathbf{F}\right)^{-1} \mathbf{F}^{\mathrm{H}} \mathbf{X}^{\mathrm{H}}(n) \underline{\mathbf{y}}_{m_{R}}(n),
$$

where $\mathbf{X}$ contains the pilot symbols or if used in a DD mode, the symbol decisions. The calculation of the LS channel estimate from the pilot symbols is simple as the matrix inversion can be calculated in advance and the only calculation to be performed in real time is multiplication with the received signal. When using the LS estimator in a DD mode, the $N L \times N L$ matrix inversion induces a high computational complexity.

\section{B. MMSE Channel Estimation}

In order to exploit the time domain correlation of the channel and to take into account the impact of the noise, the LS channel estimate can be filtered with an MMSE filter [2]

$$
\hat{\mathbf{h}}_{m_{R}, m_{T}, l}^{\mathrm{MMSE}}(n)=\mathbf{W}_{m_{R}, m_{T}, l}^{\mathrm{H}}(n) \hat{\mathbf{h}}_{m_{R}, m_{T}, l}^{\mathrm{LS}},
$$

where the LS channel estimate vector for the $l$ th tap from the $m_{T}$ th transmit antenna to the $m_{R}$ th receive antenna

$$
\hat{\mathbf{h}}_{m_{R}, m_{T}, l}^{\mathrm{LS}}=\left[\hat{h}_{m_{R}, m_{T}, l}^{\mathrm{LS}}\left(n_{1}\right) \ldots \hat{h}_{m_{R}, m_{T}, l}^{\mathrm{LS}}\left(n_{N_{P}}\right)\right]^{\mathrm{T}} \in \mathbb{C}^{N_{P} \times 1}
$$

contains the LS channel estimates from the duration of the filtering window. $N_{P}$ is the number of OFDM symbols with pilot symbols in a filtering window and $m_{T}=1, \ldots, N$ and $m_{R}=1, \ldots, M$ are the transmit and receive antenna indices. The filtering vector $\mathbf{W}_{m_{R}, m_{T}, l}(n)$ is defined as

$$
\mathbf{W}_{m_{R}, m_{T}, l}(n)=\boldsymbol{\Sigma}_{\hat{\mathbf{h}}_{m_{R}, m_{T}, l}^{\mathrm{LS}}}^{-1} \boldsymbol{\Sigma}_{m_{R}, m_{T}, l}^{\mathrm{H}},
$$

where the cross-covariance matrix between $h_{m_{R}, m_{T}, l(n)}$ and $\hat{\mathbf{h}}_{m_{R}, m_{T}, l}^{\mathrm{LS}}$ is

$$
\boldsymbol{\Sigma}_{m_{R}, m_{T}, l}=\left[\rho\left(n-n_{1}\right) \ldots \rho\left(n-n_{N_{P}}\right)\right] \Sigma_{\mathbf{h}_{l}}
$$


and the auto-covariance matrix is

$$
\begin{aligned}
\boldsymbol{\Sigma}_{\hat{\mathbf{h}}_{m_{R}, m_{T}, l}^{\mathrm{LS}}}= & {\left[\begin{array}{ccc}
\rho\left(n_{1}-n_{1}\right) & \cdots & \rho\left(n_{1}-n_{N_{P}}\right) \\
\vdots & \ddots & \vdots \\
\rho\left(n_{N_{P}}-n_{1}\right) & \cdots & \rho\left(n_{N_{P}}-n_{N_{P}}\right)
\end{array}\right] \Sigma_{\mathbf{h}_{m_{R}, m_{T}, l}} } \\
& +\boldsymbol{\Sigma}_{w} .
\end{aligned}
$$

The noise covariance matrix $\boldsymbol{\Sigma}_{w}=\sigma^{2} \mathbf{I} \in \mathbb{R}^{N_{P} \times N_{P}}$, $\Sigma_{\mathbf{h}_{m_{R}, m_{T}, l}}=E\left(h_{m_{R}, m_{T}, l}^{*} h_{m_{R}, m_{T}, l}\right)$ and $\rho\left(n-n^{\prime}\right)$ is the temporal correlation between the channel taps at times $n$ and $n^{\prime}$ [8]. In order to avoid the calculation of the spatial correlation $\Sigma_{\mathbf{h}_{l}}$, it can be left out from (6) and (7). This only has a minor impact on the performance as presented in Section IV. It also enables the use of precalculated MMSE filter coefficients, where the predetermined values for $\sigma^{2}$ and user velocity are used. The coefficients can be calculated for a set of $\sigma^{2}$ and velocity values and the coefficients closest to the estimated values can be used. Since $\rho(n-n)=1$ and $\boldsymbol{\Sigma}_{w}$ contains $\sigma^{2}$ on its diagonal, the precalculated coefficients can be obtained by substituting the known values in (5). The MMSE filter coefficients can be then precalculated as

$$
\left[\begin{array}{ccc}
1+\sigma^{2} & \cdots & \rho\left(n_{1}-n_{N_{P}}\right) \\
\vdots & \ddots & \vdots \\
\rho\left(n_{N_{P}}-n_{1}\right) & \cdots & 1+\sigma^{2}
\end{array}\right]^{-1}\left[\begin{array}{c}
\rho\left(n-n_{1}\right) \\
\vdots \\
\rho\left(n-n_{N_{P}}\right)
\end{array}\right]
$$

where the temporal correlation is distributed according to Jake's model and can be written as

$$
\rho\left(n-n^{\prime}\right)=J_{0}\left(2 \pi f_{d}\left(n-n^{\prime}\right) T_{B}\right)
$$

and $J_{0}$ denotes the zeroth-order Bessel function of the first kind, $f_{d}$ is the Doppler frequency and $T_{B}$ is the OFDM symbol duration.

\section{SAGE Channel Estimation}

The EM algorithms consist of an expectation and a maximization step. The "complete" data is estimated in the expectation step and the channel estimate is updated in the maximization step. The frequency domain SAGE algorithm provides an iterative solution of the decision directed LS estimate in (2). The time domain SAGE algorithm [12] can be used to avoid the matrix inversion required with non-constant envelope modulations in FD SAGE channel estimator. The time domain received signal $\mathbf{o}$ is viewed as the "incomplete" data and $\mathbf{z}$ as the "complete" data, which is iteratively updated along with the channel estimate $\hat{h}_{m_{T}, m_{R}, l}(n)$. The time domain SAGE algorithm calculates the channel estimates with iterations

$$
\begin{gathered}
\hat{\mathbf{z}}_{m_{T}, m_{R}, l}^{(i)}=\underline{\hat{\mathbf{z}}}_{m_{T}, m_{R}, l}^{(i)}+\left[\mathbf{o}_{m_{R}}-\sum_{m_{T}^{\prime}=1}^{M_{T}} \sum_{l^{\prime}=0}^{L-1} \underline{\hat{\mathbf{z}}}_{m_{T}^{\prime}, m_{R}, l^{\prime}}^{(i)}\right] \\
\hat{h}_{m_{T}, m_{R}, l}^{(i+1)}(n)=\frac{\overline{\mathbf{x}}_{m_{T}, l}^{\mathrm{H}} \hat{\mathbf{z}}_{m_{T}, m_{R}}^{(i)}(n)}{\overline{\mathbf{x}}_{m_{T}, l}^{\mathrm{H}} \overline{\mathbf{x}}_{m_{T}, l}} \\
\underline{\hat{\mathbf{z}}}_{m_{T}, m_{R}, l}^{(i+1)}(n)=\overline{\mathbf{x}}_{m_{T, l}} \hat{h}_{m_{T}, m_{R}, l}^{(i+1)} \\
\underline{\hat{\mathbf{z}}}_{m_{T}^{\prime \prime}, m_{R}, l^{\prime \prime}}^{(i+1)}(n)=\underline{\hat{\mathbf{z}}}_{m_{T}^{\prime \prime}, m_{R}, l^{\prime \prime}}^{(i)}
\end{gathered}
$$

The channel estimator is initialized with the channel estimate $\hat{h}_{m_{T}, m_{R}, l}^{(0)}$ from the previous OFDM symbol as

$$
\underline{\hat{\mathbf{z}}}_{m_{T}, m_{R}, l}^{(0)}(n)=\overline{\mathbf{x}}_{m_{T, l}} \hat{h}_{m_{T}, m_{R}, l}^{(0)} .
$$

\section{PERFORMANCE COMPARISON}

The vehicular channel model from [13] was used. The simulator for the MIMO fading channel model was introduced in [14] and it includes temporal, spatial and spectral correlation. Turbo coding with $1 / 2$ code rate was used in the simulations. A $2.4 \mathrm{GHz}$ carrier frequency was assumed with a $71.4 \mu \mathrm{s}$ OFDM symbol duration. A $5 \mathrm{MHz}$ system bandwidth was applied. The detector used in the simulations is a $K$-best list sphere detector (LSD) [15] with list size 16. Turbo coding was performed over one OFDM symbol. The user velocity of $50 \mathrm{~km} / \mathrm{h}$ was assumed where the corresponding Doppler frequency is $111 \mathrm{~Hz}$.

The throughput can be increased by using half of the LTE reference signals along with the decision directed channel estimation. The pilot symbols are then transmitted only in the first OFDM symbol in a slot and data is transmitted instead of pilot symbols in the other OFDM symbols which is denoted in the figures as 1 pilot. Channel estimation can be performed over several slots but this decreases the performance in high velocity scenarios.

The LS channel estimation is used on the OFDM symbols with pilot symbols and the SAGE algorithm is used on the OFDM symbols without reference signals. The performance of the SAGE algorithm with transmitted data as the feedback, i.e, genie aided SAGE, is also shown in the figure. With the genie aided mode, pilots are transmitted in one OFDM symbol per slot where the LS estimator is used in estimating the channel. The MMSE channel estimator is precalculated with the velocity of $70 \mathrm{~km} / \mathrm{h}$ and the SNR of $26.5 \mathrm{~dB}$. MMSE filtering is performed over one slot with the LTE pilot structure and over two slots with pilots in only one OFDM symbol as the MMSE filter needs at least two channel estimates to perform well. The filtering window is shifted when a new channel estimate is available from the LS estimator in order to obtain the most current estimates. Increasing the size of the filtering window with LTE pilot structure does not improve the performance.

The performance of the channel estimation algorithms is presented in Fig. 2 with a $4 \times 4$ antenna system. The communication system performance is usually characterized by frame error rate (FER). The transmission throughput is defined to be equal to the nominal information transmission rate of information bits times (1-FER). With pilot symbols in one OFDM symbol in a slot, denoted as 1 pilot, pilot symbols for all antennas are transmitted in the 1st OFDM symbol. The MMSE and SAGE estimators are able to compensate for the performance loss from the decreased pilot symbol density, unlike the LS estimator. The performance of the MMSE estimator is almost as good as with the SAGE estimator with the LTE pilot structure but when the pilot density is decreased, the performance difference is larger.

Transmitting pilot symbols for all four antennas in the $1 \mathrm{st}$ OFDM symbol in a slot improves the SAGE performance 


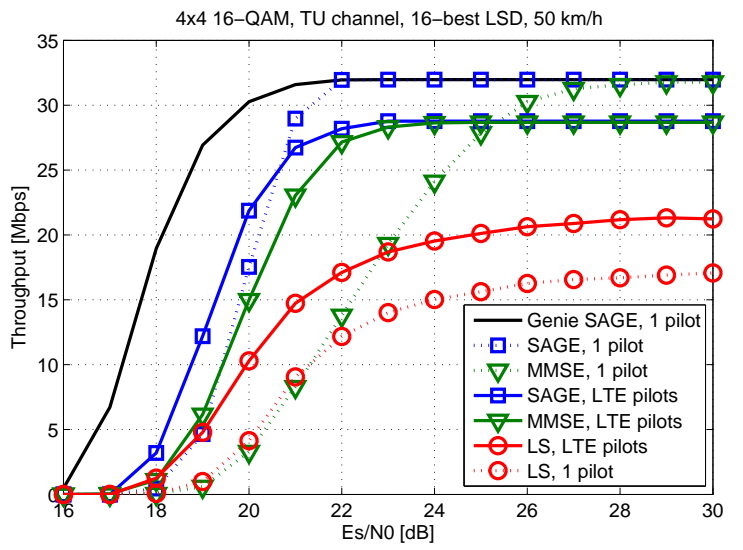

Fig. 2. $4 \times 4$ 16-QAM data transmission throughput vs. SNR with different pilot densities and $50 \mathrm{~km} / \mathrm{h}$ user velocity.

especially with high user velocities because there is no need to combine the channel estimate of the two antennas in the current OFDM symbol with the channel estimate of the other two antennas from the previous OFDM symbol. The SAGE estimator also gets a better initial guess of the channel when all pilot symbols are transmitted in the same slot and is able to estimate the channel well in the decision directed mode. With the LTE pilots, the MMSE filter performs well. However, the performance of the MMSE estimator degrades when the pilot symbol density decreases, i.e., the MMSE estimator needs a sufficient pilot symbol density to perform well. The MMSE estimator cannot be used effectively to improve the throughput by transmitting less pilots as the SAGE channel estimator.

\section{IMPLEMENTATION OF LS, MMSE AND SAGE CHANNEL ESTIMATION}

\section{A. Architecture and Memory Requirements}

The LS channel estimator includes complex multiplications of the LS coefficients and the received data symbols when performed from the pilot symbols. The calculation of each $L N M$ channel coefficient includes $P / N$ complex multiplications after which the results are added together. For a $2 \times 2$ antenna system, there are 80 channel coefficients to be calculated. If the channel estimate can be obtained with the delay of one OFDM symbol, the latency for the calculation of one channel coefficient is $0.88 \mu \mathrm{s}$.

The MMSE channel estimator consists of multiplications of the LS channel estimates with real valued coefficients. Each MMSE channel estimate coefficient $\hat{\mathbf{h}}_{m_{R}, m_{T}, l}^{\mathrm{MMSE}}$ is a composite of the $N_{P}$ LS estimates from the filtering period. With the $4 \times 4$ system, $N_{P}=3$ and the filtering period is 7 OFDM symbols. The MMSE channel estimator then performs 6 multiplications and 4 additions for each complex valued channel coefficient.

The architecture of the SAGE channel estimator for a $2 \times 2$ antenna system is presented in Fig 3. Each block corresponds to (10)-(14). The elements of $\overline{\mathbf{x}}_{m_{T}}$ in each stream are squared and the results are added together in the symbol multiplication part. The inverses of the results are multiplied with $\overline{\mathbf{x}}_{m_{T}}$. These calculations from (11) can be performed separately from the iterative channel tap calculations. For each channel tap,
$N_{c}$ iterations are performed. The channel tap iterations are initialized by multiplying the symbol decisions $\overline{\mathbf{x}}_{m_{T}}$ with the channel taps from the previous OFDM symbol in the block corresponding to (12). In later iterations, the channel taps from previous iterations are used. $L$ multiplication results from $N$ layers corresponding to the channel to each receive antenna are added together and subtracted from the received symbol from each receive antenna. The result is then added to the first $\overline{\mathbf{x}}_{m_{T}} \hat{h}_{m_{T} m_{R}}$ multiplication result in the block corresponding to (10) and multiplied with $\mathbf{x}_{i}^{-1}$ in the block corresponding to (11). A channel tap is obtained by adding together the results from the $N_{c}$ iterations. The total number of iterations in calculating all the channel taps is $M N L S_{i} N_{c}$.

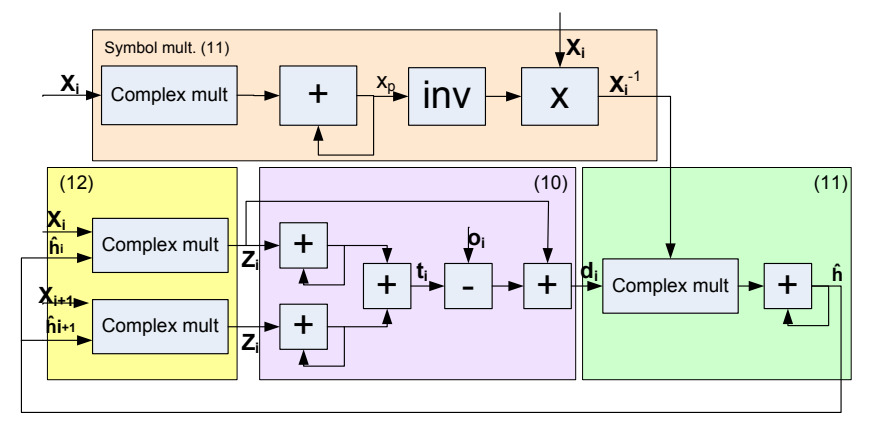

Fig. 3. The architecture of the SAGE channel estimator.

The precision of the variables required to sustain the performance close to that of the floating point variables were determined. The word lengths were determined with computer simulations using the same parameters as those in Section IV. The performance of the fixed point channel estimators compared to those of the floating point estimators are presented in Figure 4. Some performance degradation is allowed in the fixed point estimators in order to keep the complexity low as reaching the floating point performance may require a considerable increase in the word lengths. The word lengths for the SAGE estimator are from 8 to 18 bits. The LS coefficients are the precalculated results of $\left(\mathbf{F}^{H} \mathbf{X}^{H}(n) \mathbf{X}(n) \mathbf{F}\right)^{-1} \mathbf{F}^{H} \mathbf{X}^{H}(n)$ from pilot symbols. The coefficients had a word length of 12 bits while the channel estimates were 12 bits and the received signal vector was 16 bits. The MMSE coefficients were defined to be 13 bits and the results from MMSE filtering were 14 bits.

The amount of memory required to store the LS coefficients precalculated from the pilot symbols is $14.4 \mathrm{kbit}$ assuming that the pilot symbols are the same in each OFDM symbol. The highest amount of memory in the MMSE filter is needed in storing the LS channel estimates from $N_{P}$ OFDM symbols. The required amount of memory is 17.5 kbit in the $4 \times 4$ antenna system. In the SAGE channel estimator, the memory requirement for the symbol expectations $\overline{\mathbf{x}}_{i}$ is $16.4 \mathrm{kbit}$ in the $2 \times 2$ antenna system and $32.8 \mathrm{kbit}$ in the $4 \times 4$ antenna system. The highest amount of memory in the SAGE estimator would be the 1.2 Mbit for storing the interim results for $\hat{\mathbf{z}}_{i}$ but this is partly included in the following implementation results unlike the previously discussed memory requirements for the LS and MMSE filters. 


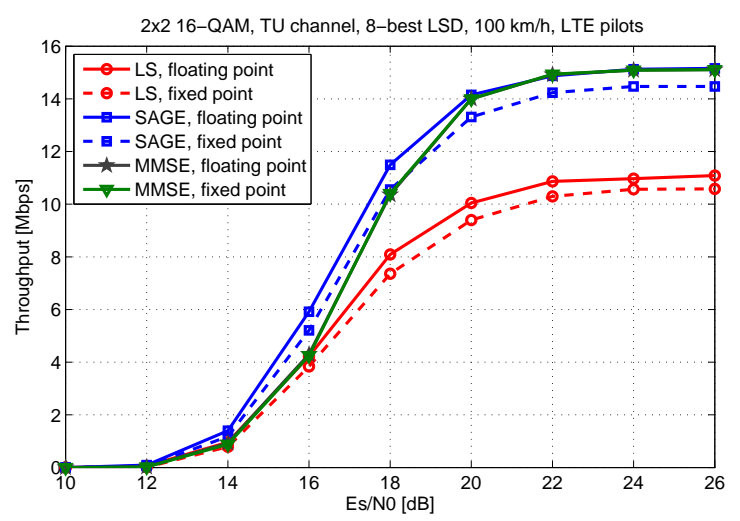

Fig. 4. $2 \times 2$ 16-QAM data transmission throughput vs. SNR with fixed word lengths.

\section{B. Implementation Results}

Catapult (B) C Synthesis tool [16] was used in the implementation of the receivers. It synthesizes algorithms written in ANSI C++ and SystemC into hardware. The Synopsys Design Compiler was used in synthesizing the VHDL along with the UMC $0.18 \mu \mathrm{m}$ CMOS technology. The FFTs are not included in the complexity estimations. The complexity and power consumption of each channel estimator is comparable only to other channel estimators presented in this paper as the results depend on the used implementation method and library. However, a few other channel estimation implementations from the literature will be briefly discussed.

The implementation results for the LS channel estimator and MMSE filter are presented in Table I with different processing times. The impact of the delay in the estimators on their performance was discussed in [11]. The estimators were implemented for $2 \times 2$ and $4 \times 4$ antenna systems and for a $5 \mathrm{MHz}$ bandwidth. The processing time of $71 \mu \mathrm{s}$ corresponds to the case of delay in channel estimates and the shorter processing time corresponds to the no delay case. The detector latency was assumed to be half from the $71 \mu$ s OFDM symbol duration but the detector itself is not included in the complexity estimates. The LS estimator latency can then be $38 \mu \mathrm{s}$ in the $2 \times 2$ antenna system and $33 \mu \mathrm{s}$ in the $4 \times 4$ antenna system. The decoder latency [17] is also included in the latency calculations and each codeword is assumed to be mapped to a single slot and not interleaved over multiple slots. The gate count increases with the bandwidth in the LS estimator and the results can be scaled to higher bandwidths. The longer processing delay does not have a major impact on the complexity but the power consumption can be decreased.

The implementation results for the SAGE channel estimator are presented in Table II. There are two target processing times for the SAGE estimator. The SAGE channel estimator has 31 $\mu \mathrm{s}$ in the no delay case to calculate the channel estimates in the $2 \times 2$ antenna system when the detector latency, the decoder latency of $5 \mu \mathrm{s}$, the symbol expectation latency of 1 $\mu \mathrm{s}$ and the IFFT [18] latency of $1 \mu \mathrm{s}$ is subtracted from the OFDM symbol time. The symbol expectation calculation was implemented with the architecture from [19] and the gate count of $5.5 \mathrm{k}$ gates was added to the SAGE complexity in Table
TABLE I

SYNTHESIS RESULTS FOR THE LS AND MMSE CHANNEL ESTIMATORS WITH DIFFERENT LATENCIES

\begin{tabular}{|c|c|c|c|c|}
\hline & LS & MMSE & LS & MMSE \\
\hline & \multicolumn{2}{|c|}{ No delay } & \multicolumn{2}{|c|}{ Delay } \\
\hline & \multicolumn{4}{|c|}{$2 \times 2$} \\
\hline Processing time & $38 \mu \mathrm{s}$ & $38 \mu \mathrm{s}$ & $71 \mu \mathrm{s}$ & $71 \mu \mathrm{s}$ \\
\hline Clock frequency & $95 \mathrm{MHz}$ & $95 \mathrm{MHz}$ & $51 \mathrm{MHz}$ & $51 \mathrm{MHz}$ \\
\hline Equivalent gates & 3763 & 3213 & 3730 & 3213 \\
\hline \multirow[t]{2}{*}{ Power consumption } & $8.3 \mathrm{~mW}$ & $5.2 \mathrm{~mW}$ & $4.8 \mathrm{~mW}$ & $2.8 \mathrm{~mW}$ \\
\hline & \multicolumn{4}{|c|}{$4 \times 4$} \\
\hline Processing time & $33 \mu \mathrm{s}$ & $33 \mu \mathrm{s}$ & $71 \mu \mathrm{s}$ & $71 \mu \mathrm{s}$ \\
\hline Clock frequency & $146 \mathrm{MHz}$ & $146 \mathrm{MHz}$ & $102 \mathrm{MHz}$ & $68 \mathrm{MHz}$ \\
\hline Equivalent gates & 3759 & 4549 & 3763 & 4375 \\
\hline Power consumption & $13.2 \mathrm{~mW}$ & $10.2 \mathrm{~mW}$ & $8.9 \mathrm{~mW}$ & $4.6 \mathrm{~mW}$ \\
\hline
\end{tabular}

II. In the $4 \times 4$ antenna system, the decoder and IFFT have higher latencies and the processing time for SAGE channel estimation is only $25 \mu \mathrm{s}$.

The longer processing times of 64 and $59 \mu$ s correspond to the case when channel estimation is performed during the detection of the following OFDM symbol. The IFFT complexity was not considered in the total complexity. An IFFT block could be added to the receiver or the FFT with scaling could be reused, timing permitting. The LS estimator would be included in the receiver with SAGE channel estimator and the complexity and power estimates would have to be added together to get the total complexity of the SAGE channel estimator.

TABLE II

SYNTHESIS RESULTS FOR THE SAGE CHANNEL ESTIMATOR WITH DIFFERENT LATENCIES

\begin{tabular}{|l|l|l|}
\hline & Delay & No delay \\
\hline & \multicolumn{2}{|c|}{$2 \times 2$} \\
\hline Processing time & $64 \mu \mathrm{s}$ & $31 \mu \mathrm{s}$ \\
Symbols, $S_{i}$ & $1 / 2,3$ & $1 / 2,4$ \\
Clock frequency & $144 \mathrm{MHz}$ & $165 \mathrm{MHz}$ \\
Equivalent gates & $34.6 \mathrm{k}$ & $58.2 \mathrm{k}$ \\
Power consumption & $93 \mathrm{~mW}$ & $189 \mathrm{~mW}$ \\
\hline & \multicolumn{2}{|c|}{$4 \times 4$} \\
\hline Processing time & $59 \mu \mathrm{s}$ & $25 \mu \mathrm{s}$ \\
Symbols, $S_{i}$ & $1 / 4,4$ & $1 / 4,4$ \\
Clock frequency & $104 \mathrm{MHz}$ & $147 \mathrm{MHz}$ \\
Equivalent gates & $113.4 \mathrm{k}$ & $210 \mathrm{k}$ \\
Power consumption & $257 \mathrm{~mW}$ & $604 \mathrm{~mW}$ \\
\hline
\end{tabular}

Implementation results for channel estimators have not been presented extensively in the literature. The approximate linear MMSE channel estimator from [9] uses the noise and correlation in calculating the coefficients. The implementation cost is $49 \mathrm{k}$ gates but the algorithm is different from the MMSE in this paper making a comparison difficult. Data carriers are exploited in channel estimation for calculating channel variations in [10]. The algorithm provides better performance in fast fading scenarios but the complexity of the channel estimator is $1901 \mathrm{k}$ gates. Furthermore, the implementation was done for a wireless local area network (WLAN) system.

The LS estimator is used throughout the paper for obtaining the initial channel estimates from pilot symbols and the SAGE is used for updating the channel estimates. The use of DD LS estimation would be prohibitive because of the high complexity as stated in Section IV. With higher bandwidths 
or numbers of antennas, the time in which the SAGE channel estimation should be performed is shorter due to the increased decoder and FFT latencies. This results in higher complexity and power consumption. In terms of throughput per number of gates, the pilot only LS estimator uses the least number of gates per bit. However, in higher velocities, the MMSE and SAGE estimators would greatly improve the performance. Furthermore, the throughput can be increased by decreasing the pilot symbol density and using the SAGE estimator in calculating the channel estimates.

The energy efficiency of the pilot based LS, MMSE and the DD SAGE channel estimators is presented in Table III. The throughput is achieved in a $4 \times 4$ antenna system at 22 $\mathrm{dB}$ with $50 \mathrm{~km} / \mathrm{h}$ user velocity. The throughput with perfect channel state information would be $32 \mathrm{Mb} / \mathrm{s}$ with pilot symbols in one OFDM symbol per slot. The power consumption of the LS estimator is included in all the estimators. The MMSE estimator with processing delay has the best energy efficiency but the SAGE estimator with delay can be used for improved throughput. When using the SAGE channel estimator at the receiver, less transmit power is needed for achieving the required throughput.

TABLE III

LS, MMSE AND SAGE ENERGY EFFICIENCY COMPARISON

\begin{tabular}{|l|l|l|l|l|}
\hline Estimator & Pilots & Delay & Throughput & Energy/bit \\
\hline LS & LTE & No & $17.1 \mathrm{Mb} / \mathrm{s}$ & $0.77 \mathrm{~nJ} / \mathrm{b}$ \\
LS & LTE & Yes & $11.6 \mathrm{Mb} / \mathrm{s}$ & $0.767 \mathrm{~nJ} / \mathrm{b}$ \\
MMSE & LTE & No & $27.2 \mathrm{Mb} / \mathrm{s}$ & $0.86 \mathrm{~nJ} / \mathrm{b}$ \\
MMSE & LTE & Yes & $19.6 \mathrm{Mb} / \mathrm{s}$ & $0.388 \mathrm{~nJ} / \mathrm{b}$ \\
SAGE & 1 pilot & Yes & $31.2 \mathrm{Mb} / \mathrm{s}$ & $8.6 \mathrm{~nJ} / \mathrm{b}$ \\
\hline
\end{tabular}

\section{CONCLUSIONS}

The performance of the DD SAGE channel estimation with the possibility of using it to improve the performance from the pilot symbol based estimators was considered. The least squares estimator was used in obtaining the channel estimates from pilot symbols. Time domain correlation of the channel estimates was exploited in the MMSE filter when calculating estimates for symbols with no pilots. The implementation results for the pilot based LS estimator, the SAGE channel estimator and the MMSE filter were presented.

The complexity and power consumption of the LS and MMSE estimators are low. The delay after which the channel estimates from SAGE are available for detection has a high impact on the complexity and performance. The complexity and power consumption can be high when using the SAGE estimator with a short processing delay. A good performancecomplexity trade-off can be achieved by allowing a longer processing delay for the SAGE estimator.

The MMSE filter and the SAGE estimator improve the pilot symbol based LS performance with high user velocities when the channel changes frequently between pilot symbols. The throughput can be increased by decreasing the pilot symbol density and transmitting data instead of pilot symbols. The SAGE estimator can then be used in calculating channel estimates when pilot symbols are not transmitted. The SAGE channel estimator would be a good choice for systems where training is performed in the beginning of the transmission or less frequently. The MMSE estimator is suitable for systems with high pilot densities.

\section{REFERENCES}

[1] 3rd Generation Partnership Project (3GPP); Technical Specification Group Radio Access Network, "Evolved universal terrestrial radio access E-UTRA; physical channels and modulation (release 10) TS 36.211 (version 10.0.0)," Tech. Rep., 2010.

[2] S. M. Kay, Fundamentals of Statistical Signal Processing: Estimation Theory. Englewood Cliffs, NJ, USA: Prentice-Hall, 1993.

[3] M. Morelli and U. Mengali, "A comparison of pilot-aided channel estimation methods for OFDM systems," IEEE Trans. Signal Processing, vol. 49, no. 12, pp. 3065-3073, Dec. 2001.

[4] I. Barhumi, G. Leus, and M. Moonen, "Optimal training design for MIMO-OFDM systems in mobile wireless channels," IEEE Trans. Signal Processing, vol. 51, no. 6, pp. 1615-1624, Jun. 2003.

[5] A. P. Dempster, N. M. Laird, and D. B. Rubin, "Maximum likelihood from incomplete data via the EM algorithm," J. Royal Stat. Soc., vol. 39, no. 1, pp. 1-38, 1977.

[6] Y. Xie and C. N. Georghiades, "Two EM-type channel estimation algorithms for OFDM with transmitter diversity," IEEE Trans. Commun., vol. 51, no. 1, pp. 106-115, Jan. 2003.

[7] J.-J. van de Beek, O. Edfors, M. Sandell, S. Wilson, and P. Borjesson, "On channel estimation in OFDM systems," in Proc. IEEE Veh. Technol. Conf., vol. 2, Chicago, IL, USA, Jul. 25-28 1995, pp. 815-819.

[8] H. Miao and M. Juntti, "Space-time channel estimation and performance analysis for wireless MIMO-OFDM systems with spatial correlation," IEEE Trans. Veh. Technol., vol. 54, no. 6, pp. 2003-2016, Nov. 2005.

[9] M. Simko, D. Wu, C. Mehlfuehrer, J. Eilert, and D. Liu, "Implementation aspects of channel estimation for 3GPP LTE terminals," in Proc. European Wireless Conf., Vienna, Austria, Apr.27-29 2011.

[10] M.-F. Sun, T.-Y. Juan, K.-S. Lin, and T.-Y. Hsu, "Adaptive frequencydomain channel estimator in $4 \times 4$ MIMO-OFDM modems," IEEE Trans. VLSI Syst., vol. 17, no. 11, pp. 1616 -1625, Nov. 2009.

[11] J. Ketonen, M. Juntti, and J. Ylioinas, "Decision directed channel estimation for reducing pilot overhead in LTE-A," in Proc. Annual Asilomar Conf. Signals, Syst., Comp., Pacific Grove, USA, Nov.7-10 2010, pp. 1503-1507.

[12] J. Ylioinas, M. R. Raghavendra, and M. Juntti, "Avoiding matrix inversion in DD SAGE channel estimation in MIMO-OFDM with MQAM," in Proc. IEEE Veh. Technol. Conf., Anchorage, USA, Sep. 20-23 2009 , pp. $1-5$.

[13] 3rd Generation Partnership Project (3GPP); Technical Specification Group Radio Access Network, "Spatial channel model for multiple input multiple output (MIMO) simulations (3G TS 25.996 version 6.0.0 (release 6))," 3rd Generation Partnership Project (3GPP), Tech. Rep., 2003.

[14] E. Kunnari and J. Iinatti, "Stochastic modelling of Rice fading channels with temporal, spatial and spectral correlation," IET Communications, vol. 1, no. 2, pp. 215-224, Apr. 2007.

[15] K. Wong, C. Tsui, R. K. Cheng, and W. Mow, "A VLSI architecture of a K-best lattice decoding algorithm for MIMO channels," in Proc. IEEE Int. Symp. on Circuits and Systems, vol. 3, Scottsdale, AZ, May 26-29 2002, pp. 273-276.

[16] Mentor Graphics, "Catapult C Synthesis Overview, Tech. Rep., http://www.mentor.com/esl/catapult/overview," 2011.

[17] Y. Sun, Y. Zhu, M. Goel, and J. Cavallaro, "Configurable and scalable high throughput turbo decoder architecture for multiple $4 \mathrm{G}$ wireless standards," in IEEE Int. Conf. on Application-specific Systems, Architectures and Processors (ASAP), Leuven, Belgium, Jul.2-4 2008, pp. 209-214.

[18] K. Babionitakis, K. Manolopoulos, K. Nakos, and V. Chouliaras, "A high performance VLSI FFT architecture," in IEEE International Conference on Electronics, Circuits and Systems, Nice, France, Dec. 10-13 2006, pp. 810-813.

[19] J. Ketonen, M. Juntti, and J. Cavallaro, "Performance-complexity comparison of receivers for a LTE MIMO-OFDM system," IEEE Trans. Signal Processing, vol. 58, no. 6, pp. 3360-3372, Jun. 2010. 